\title{
Sugar Mill Effluent Utilization in the Cultivation of Maize (Zea mays L.) in Two Seasons
}

\begin{abstract}
Vinod Kumar
Agro-Ecology and Pollution Research Laboratory, Department of Zoology and Environmental Science, Gurukula Kangri University, Haridwar, Uttarakhand 249404, India

Correspondence should be addressed to Vinod Kumar; drvksorwal@gmail.com

Received 28 May 2014; Revised 30 August 2014; Accepted 31 August 2014; Published 23 September 2014

Academic Editor: Dimitris P. Makris

Copyright (C) 2014 Vinod Kumar. This is an open access article distributed under the Creative Commons Attribution License, which permits unrestricted use, distribution, and reproduction in any medium, provided the original work is properly cited.

The aim of present investigation is to study the effects of sugar mill effluent fertigation on soil properties and agronomical characteristics of Maize (Zea mays L. cv. NMH 589) in two seasons. Six treatments of sugar mill effluent, namely, 0\% (control), $20 \%, 40 \%, 60 \%, 80 \%$, and $100 \%$, were used for the cultivation of $Z$. mays. Fertigation with different concentrations of sugar mill effluent resulted in significant $(P<0.01)$ changes in $\mathrm{EC}, \mathrm{pH}, \mathrm{OC}, \mathrm{Na}^{+}, \mathrm{K}^{+}, \mathrm{Ca}^{2+}, \mathrm{Mg}^{2+}, \mathrm{TKN}, \mathrm{PO}_{4}{ }^{3-}, \mathrm{SO}_{4}{ }^{2-}, \mathrm{Fe}^{3+}, \mathrm{Zn}^{2+}, \mathrm{Cd}^{2+}, \mathrm{Cu}^{2+}$, $\mathrm{Mn}^{2+}$, and $\mathrm{Cr}^{3+}$ of the soil in both seasons. The maximum agronomic performance of $Z$. mays was noted with $40 \%$ concentration of sugar mill effluent. Biochemical components like crude proteins, crude fiber, and total carbohydrates were recorded highest with $40 \%$ concentration of sugar mill effluent in both seasons. The contamination factor (Cf) of various heavy metals was observed in order of $\mathrm{Mn}^{2+}>\mathrm{Zn}^{2+}>\mathrm{Cu}^{2+}>\mathrm{Cd}^{2+}>\mathrm{Cr}^{3+}$ for soil and $\mathrm{Mn}^{2+}>\mathrm{Zn}^{2+}>\mathrm{Cu}^{2+}>\mathrm{Cr}^{3+}>\mathrm{Cd}^{2+}$ for Z. mays in both seasons after fertigation with sugar mill effluent. It appears that sugar mill effluent can be used as a biofertigant after appropriate dilution to improve the yield.
\end{abstract}

\section{Introduction}

In many agroindustrial processes, some by-products may be utilized as useful products $[1,2]$. In India, the sugar industry is one of the greatest industries, based on sugar cane. Presently India has nearly 650 sugar mills that produce about 15 million tons of sugar and 13 million tons of molasses annually $[3,4]$. The high production of sugar generates high quantities of the sugar industry wastes such as effluent, filter mud cake, vinasse, molasses, bagasse, and bagasse ash [5]. A few years ago, these by-products were considered as a waste and were often disposed of causing environmental problems such as aquatic and terrestrial pollution [6]. Recently, it has been recognized that such by-products should be considered as useful materials [7]. These by-products are of great agricultural interest because of their high organic matter, $\mathrm{N}$ and $\mathrm{K}$ contents, and probably other elements $[3,8,9]$. Therefore, some of these sugar industry by-products may represent an important source of nutrients and thereby could be used as a substitute for chemical and organic fertilizers $[2,8,10,11]$.
Use of wastewater in agricultural fields may be a viable method of disposal and would sustain agriculture in nonirrigated areas where the availability of fresh water is scarce $[12,13]$. It reduces fertilizer and irrigation water cost as it is available without paying any cost and rich in various plant nutrients [12]. Although, metals like $\mathrm{Cu}, \mathrm{Fe}, \mathrm{Ni}$, and $\mathrm{Zn}$ and other trace elements are important for proper functioning of biological systems, and their deficiency, or excess could lead to a number of disorders [14-16]. But long term irrigation with effluents increases accumulation of metals in soil and plants and increases chances of their entrance in food chain [17]. Contamination of agricultural soils with metals can pose long term environmental problems and is not without health implications [17-19].

Maize (Zea mays L.) is the most important grain crop and is cultivated for food and fodder throughout the world under diverse environments [20, 21]. Maize biomass is variously used for the production of energy, fiber, or paper, as well as for syrup and animal feed [22, 23]. In India, it ranks fourth after rice, wheat, and sorghum. It is cultivated for 
food and fodder in the northwestern states of the country in all the seasons, namely, rainy, winter, and summer, with a production of 14.71 million tonnes from an area of 7.23 million hectares with productivity of 19.04 quintals per hectare [4]. It also has the ability to tolerate and survive under adverse conditions of intermittent and continuing drought [24, 25]. Therefore, maize has received considerable attention during the last years as an alternative source for food, fodder, and energy production $[4,11]$.

Some crops have higher potential yields with wastewater irrigation, reduce the need for chemical fertilizers, and result in net cost savings to farmers $[13,26]$. It is important to understand the specificity of crop-effluent interaction for appropriate applications in irrigation [13]. In some studies characteristics of the effluent of industries and agronomic properties of various crop plants have been determined [10, 27-31]. Most studies were conducted on few agronomic stages with limited parameters in various crops, but there are few reports on comprehensive agronomic studies at various agronomic stages of these plants [26]. Use of industrial effluents on cultivation of $Z$. mays is receiving attention [4] but additional information is needed on how this crop responds to various concentrations of different types of effluents. The investigation was undertaken to study the effects of sugar mill effluent fertigation on soil properties and agronomical characteristics of $Z$. mays in two seasons.

\section{Materials and Methods}

2.1. Experimental Design. A field study was conducted at the Experimental Garden of the Department of Zoology and Environmental Sciences, Faculty of Life Sciences, Gurukula Kangri University, Haridwar, India $\left(29^{\circ} 55^{\prime} 10.81^{\prime \prime} \mathrm{N}\right.$ and $78^{\circ} 07^{\prime} 08.12^{\prime \prime} \mathrm{E}$ ), to determine the effects of sugar mill effluent fertigation on $Z$. mays. Six plots (each plot had an area of $9 \mathrm{~m}^{2}$ ) were selected for six treatments of sugar mill effluent, namely, $0 \%$ (control), $20 \%, 40 \%, 60 \%, 80 \%$, and $100 \%$, for the cultivation of $Z$. mays. The six treatments were placed within randomized complete block design.

2.2. Sowing of Seeds of $Z$. mays. Seeds of $Z$. mays were sown at the end of April 2010 and 2011 for the summer season crop and at the end of July 2010 and 2011 for the rainy season crop. Seeds of $Z$. mays, cv. NMH 589, were procured from Indian Council of Agriculture Research (ICAR), Pusa, New Delhi, and sterilized with $0.01 \%$ mercuric chloride and soaked in water for 12 hours. Seeds were sown in 10 rows with a distance of $30.0 \mathrm{~cm}$ between rows, while distance between the seeds was $15 \mathrm{~cm}$. The thinning was done manually after 15 days of germination to maintain the desired plant spacing and to avoid competition between plants.

2.3. Effluent Collection and Analysis. The effluent samples were collected from the R.B.N.S. Sugar Mill, Laksar, Haridwar, Uttarakhand, India $\left(29^{\circ} 44^{\prime} 46^{\prime \prime} \mathrm{N} 78^{\circ} 1^{\prime} 46^{\prime \prime} \mathrm{E}\right)$, which produces sugar from sugar cane at the rate of 150 ton sugar per day. The effluent was collected in the plastic container from the outlet of the settling tank situated in the campus of the sugar mill to reduce the biological oxygen demand (BOD) and solids from the effluent. The effluent was brought to the laboratory and then analyzed for total dissolved solids (TDS), $\mathrm{pH}$, electrical conductivity (EC), dissolved oxygen (DO), BOD, chemical oxygen demand (COD), chlorides $\left(\mathrm{Cl}^{-}\right)$, bicarbonates $\left(\mathrm{HCO}_{3}{ }^{-}\right)$, carbonates $\left(\mathrm{CO}_{3}{ }^{2-}\right)$, sodium $\left(\mathrm{Na}^{+}\right)$, potassium $\left(\mathrm{K}^{+}\right)$, calcium $\left(\mathrm{Ca}^{2+}\right)$, magnesium $\left(\mathrm{Mg}^{2+}\right)$, total Kjeldahl nitrogen (TKN), nitrate $\left(\mathrm{NO}_{3}{ }^{2-}\right)$, phosphate $\left(\mathrm{PO}_{4}{ }^{3-}\right)$, sulphate $\left(\mathrm{SO}_{4}{ }^{2-}\right)$, iron $\left(\mathrm{Fe}^{3+}\right)$, zinc $\left(\mathrm{Zn}^{2+}\right)$, cadmium $\left(\mathrm{Cd}^{2+}\right)$, copper $\left(\mathrm{Cu}^{2+}\right)$, manganese $\left(\mathrm{Mn}^{2+}\right)$, chromium $\left(\mathrm{Cr}^{3+}\right)$, standard plate count (SPC), and most probable number (MPN) following $[32,33]$ and used as fertigant.

2.4. Irrigation Pattern, Soil Sampling, and Analysis. The soil in each plot was fertigated twice in a month with 50 gallons of sugar mill effluent with $20 \%, 40 \%, 60 \%, 80 \%$, and $100 \%$ concentrations along with bore well water as the control. The soil was analyzed before sowing and after harvesting of the crop for various physicochemical parameters, namely, bulk density (BD), water holding capacity (WHC), soil texture, soil $\mathrm{pH}, \mathrm{EC}, \mathrm{OC}, \mathrm{Na}^{+}, \mathrm{K}^{+}, \mathrm{Ca}^{2+}, \mathrm{Mg}^{2+}, \mathrm{TKN}, \mathrm{PO}_{4}{ }^{3-}, \mathrm{SO}_{4}{ }^{2-}$, $\mathrm{Fe}^{3+}, \mathrm{Zn}^{2+}, \mathrm{Cd}^{2+}, \mathrm{Cu}^{2+}, \mathrm{Mn}^{2+}$, and $\mathrm{Cr}^{3+}$, following standard methods [33].

2.5. Study of Crop Parameters. The various agronomic parameters of $Z$. mays at different stages (0-90 days) were noted by standard methods for seed germination, plant height, root length, number of leaves per plant, cob length, and crop yield [34]; fresh and dry weight [35]; chlorophyll content [36]; relative toxicity (RT) [37]; leaf area index (LAI) [38]; and harvest index (HI) [39]. The nutrient quality of the crop was determined by using the following parameters: crude protein and crude fiber [40] and the total carbohydrates in dry matter were determined by the anthrone reagent method [41].

2.6. Extraction of Metals and Their Analysis. For heavy metal analysis $5 \mathrm{~mL}$ sample of sugar mill effluent and $1.0 \mathrm{~g}$ of air-dried soil or plants were digested in tubes with $3 \mathrm{~mL}$ of concentrated $\mathrm{HNO}_{3}$ digested in an electrically heated block for 1 hour at $145^{\circ} \mathrm{C}$. To this mixture $4 \mathrm{~mL}$ of $\mathrm{HClO}_{4}$ was added and heated to $240^{\circ} \mathrm{C}$ for 1 hour. The mixture was cooled and filtered through Whatman \# 42 filter paper and made with $50 \mathrm{~mL}$ and used for analysis. Heavy metals were analyzed using an atomic absorption spectrophotometer (PerkinElmer, Analyst 800 AAS, Gen Tech Scientific Inc., Arcade, NY) following APHA [32] and Chaturvedi and Sankar [33]. The contamination factor (Cf) for heavy metals accumulated in sugar mill effluent irrigated soil and $Z$. mays was calculated following Hakanson [42].

2.7. Statistical Analysis. Data were analyzed with SPSS (version 12.0, SPSS Inc., Chicago, IL). Data was subjected to two-way ANOVA. Duncan's multiple range test was also performed to determine that the difference was significant or nonsignificant. Mean standard deviation and coefficient of 
correlation ( $r$-value) of soil and crop parameters with effluent concentrations were calculated with MS Excel (version 2003, Microsoft Redmond Campus, Redmond, WA) and graphs produced with Sigma plot (version 12.3, Systat Software, Inc., Chicago, IL).

\section{Results and Discussion}

3.1. Characteristics of Sugar Mill Effluent. Values of physicochemical and microbiological parameters were different over sugar mill effluent concentration (Table 1). The sugar mill effluent was highly alkaline with a $\mathrm{pH}$ 8.98. The alkaline nature of the sugar mill effluent might be due to the presence of high concentrations of alkalis used in the sugar manufacturing process. The $\mathrm{BOD}, \mathrm{COD}, \mathrm{Cl}^{-}, \mathrm{Ca}^{2+}, \mathrm{Fe}^{3+}$, $\mathrm{TKN}, \mathrm{SO}_{4}{ }^{2-}, \mathrm{MPN}$, and SPC were beyond the prescribed limit of the Indian irrigation standards [43]. Higher BOD and COD might be due to the presence of more oxidizable organic matter and rapid consumption of dissolved inorganic materials. The higher bacterial load (SPC and MPN) in sugar mill effluent might be due to the presence of more dissolved solids and organic matter in sugar mill effluent as earlier reported by Kumar and Chopra [12]. The presence of MPN in sugar mill effluent may be due to sewage received from the toilet of staff quarters and offices. The TKN, $\mathrm{PO}_{4}{ }^{3-}, \mathrm{K}^{+}, \mathrm{Ca}^{2+}$, and $\mathrm{Mg}^{2+}$ in effluent were higher than the prescribed standards. In the present study, the values of BOD, $\mathrm{COD}, \mathrm{TKN}, \mathrm{Cl}^{-}, \mathrm{SO}_{4}{ }^{2-}$, and $\mathrm{PO}_{4}{ }^{3-}$ were more in sugar mill effluent than the values of BOD $\left(2769.00 \mathrm{mgL}^{-1}\right)$, COD $\left(4830.00 \mathrm{mgL}^{-1}\right)$, chlorides $\left(789.00 \mathrm{mgL}^{-1}\right)$, sulphate $\left(374.95 \mathrm{mgL}^{-1}\right)$, and phosphate $\left(23.00 \mathrm{mgL}^{-1}\right)$ in sugar mill effluent reported by Ezhilvannan et al. [4]. In case of metals, the contents of $\mathrm{Fe}^{3+}, \mathrm{Zn}^{2+}, \mathrm{Cd}^{2+}, \mathrm{Cu}^{2+}, \mathrm{Cr}^{3+}$, and $\mathrm{Mn}^{2+}$ were higher than the permissible limit for industrial effluent [43]. The content of these metals in sugar mill effluent was also higher than the content of $\mathrm{Zn}\left(16.34 \mathrm{mgL}^{-1}\right), \mathrm{Cd}$ $\left(3.47 \mathrm{mgL}^{-1}\right)$, and $\mathrm{Cu}\left(8.62 \mathrm{mgL}^{-1}\right)$ in sugar mill effluent reported by Borole and Patil [44].

3.2. Effects of Sugar Mill Effluent on Soil Properties. Physicochemical characteristics of the soil changed after fertigation with sugar mill effluent (Tables 2-5; Figure 1). At harvest of $Z$. mays (90 days after sowing) there was no significant change in the soil texture (loamy sand; $40 \%$ sand: $40 \%$ silt: $20 \%$ clay). Irrigation with $100 \%$ sugar mill effluent had the most increase in EC, OC, $\mathrm{Na}^{+}, \mathrm{K}^{+}, \mathrm{Ca}^{2+}, \mathrm{Mg}^{2+}, \mathrm{Fe}^{2+}$, TKN, $\mathrm{PO}_{4}{ }^{3-}, \mathrm{SO}_{4}{ }^{2-}, \mathrm{Zn}^{2+}, \mathrm{Cd}^{2+}, \mathrm{Cu}^{2+}, \mathrm{Mn}^{2+}$, and $\mathrm{Cr}^{3+}$ in both seasons (Tables 3 and 4). WHC and BD were reduced from their initial (control) values $44.36 \%$ and $1.42 \mathrm{gm} \mathrm{cm}^{-3}$ to $42.48,41.86 \%$, and $1.41 \mathrm{gm} \mathrm{cm}^{-3}$, respectively, with $100 \%$ concentration of sugar mill effluent. Seasons, sugar mill effluent concentrations and interaction of seasons, and sugar mill effluent concentrations showed insignificant $(P>0.05)$ effect on WHC and BD in both seasons (Table 2). WHC is related to the number and size distribution of soil pores, soil moisture content, textural class, structure, salt content, and organic matter. The BD of soil changes with the application of organic manure to soil that substantially modifies and lowers the soil bulk density. It is used for determining the amount of pore space and water storage capacity of the soil. Organic matter supplied through the sugar mill effluent and other kinds of wastes, like sludge, can lower the BD and WHC [45]. The findings were also in accordance with Baskaran et al. [46].

Sugar mill effluent concentrations significantly $(P<$ 0.05) affected the $\mathrm{pH}$ and $\mathrm{EC}$ of the soil. But cropping seasons showed insignificant $(P>0.05)$ effect on $\mathrm{pH}$ and EC of the soil. Seasons, sugar mill effluent concentrations, and their interaction affected the OC and TKN of the soil (Table 2). The $40 \%$ to $100 \%$ concentrations of sugar mill effluent significantly $(P<0.01)$ affected the EC, OC, $\mathrm{Na}^{+}$, $\mathrm{K}^{+}, \mathrm{Ca}^{2+}, \mathrm{Mg}^{2+}, \mathrm{TKN}, \mathrm{Fe}^{3+}, \mathrm{PO}_{4}{ }^{3-}, \mathrm{SO}_{4}{ }^{2-}, \mathrm{Zn}^{2+}, \mathrm{Cu}^{2+}$, and $\mathrm{Cr}^{3+}$ in the soil in both seasons. The $20 \%$ sugar mill effluent concentration also significantly $(P<0.05)$ affected $\mathrm{OC}$, $\mathrm{Na}^{+}, \mathrm{Ca}^{2+}, \mathrm{Mg}^{2+}, \mathrm{TKN}, \mathrm{PO}_{4}{ }^{3-}$, and $\mathrm{SO}_{4}{ }^{2-}$ in both seasons (Tables 3 and 4). Soil pH was significantly $(P<0.05)$ affected with $60 \%$ to $100 \%$ sugar mill effluent concentrations while Mn was affected by $80 \%$ and $100 \%$ sugar mill effluent concentrations (Table 4). The EC, OC, $\mathrm{Na}^{+}, \mathrm{K}^{+}, \mathrm{Ca}^{2+}, \mathrm{Mg}^{2+}$, $\mathrm{Fe}^{3+}, \mathrm{TKN}, \mathrm{PO}_{4}{ }^{3-}, \mathrm{SO}_{4}{ }^{2-}, \mathrm{Zn}^{2+}, \mathrm{Cd}^{2+}, \mathrm{Cu}^{2+}, \mathrm{Mn}^{2+}$, and $\mathrm{Cr}^{3+}$ were positively correlated with all concentrations of sugar mill effluent in both seasons (Table 5). In the present study, more irrigation of $Z$. mays with sugar mill effluent considerably increased the $\mathrm{OC}, \mathrm{Na}^{+}, \mathrm{K}^{+}, \mathrm{Ca}^{2+}, \mathrm{Mg}^{2+}, \mathrm{Fe}^{3+}$, $\mathrm{TKN}, \mathrm{PO}_{4}{ }^{3-}, \mathrm{SO}_{4}{ }^{2-}, \mathrm{Zn}^{2+}, \mathrm{Cd}^{2+}, \mathrm{Cu}^{2+}, \mathrm{Mn}^{2+}$, and $\mathrm{Cr}^{3+}$ in the soil (Table 2).

Baskaran et al. [46] reported that sugar mill effluent increased EC, pH, total organic carbon (TOC), total Kjeldahl nitrogen (TKN), available phosphorus, and exchangeable $\mathrm{Na}$, $\mathrm{K}, \mathrm{Ca}$, and $\mathrm{Mg}$ in soil. Effluent irrigation generally adds $\mathrm{PO}_{4}{ }^{3-}, \mathrm{HCO}_{3}{ }^{-}, \mathrm{Cl}^{-}, \mathrm{Na}^{+}, \mathrm{Ca}^{2+}, \mathrm{K}^{+}, \mathrm{Mg}^{2+}, \mathrm{Zn}^{2+}, \mathrm{Cd}^{2+}, \mathrm{Cr}^{3+}$, $\mathrm{Cu}^{2+}, \mathrm{Ni}^{2+}$, and $\mathrm{Mn}^{2+}$ to the soil [17]. The soil $\mathrm{pH}$ is an important parameter as many nutrients are available to plants only within a particular $\mathrm{pH}$ range. A pH range of 6.00 to 8.30 enhances nutrient availability for plants, and a $\mathrm{pH}$ below 6.00 and above 8.80 inhibits the availability of nutrients for plants [47]. In the present study, $\mathrm{pH}$ of the soil was between 8.63 and 8.72 after irrigation with $100 \%$ sugar mill effluent that may increase various soil nutrients.

Total organic matter content in the soil irrigated with effluent was higher than the soil irrigated with bore well water. The more organic matter in effluent irrigated soil might be due to the more organic nature of the effluent. Kumar and Chopra [13] found the higher organic matter in the soil irrigated with distillery effluent. Average values of TKN, $\mathrm{PO}_{4}{ }^{3-}$, and $\mathrm{K}^{+}$in the effluent irrigated soil were found to be higher than the control soil and it might be due to the higher values of TKN, $\mathrm{PO}_{4}{ }^{3-}$, and $\mathrm{K}^{+}$in the sugar mill effluent. The content of $\mathrm{Na}^{+}$and $\mathrm{SO}_{4}{ }^{2-}$ were observed higher in the soil irrigated with sugar mill effluent indicating a link between soil $\mathrm{Na}^{+}$and $\mathrm{SO}_{4}{ }^{2-}$ and higher EC in the sugar mill effluent.

Seasons, sugar mill effluent concentrations, and their interaction affected all metals like $\mathrm{Zn}, \mathrm{Cd}^{2+}, \mathrm{Cu}^{2+}, \mathrm{Mn}^{2+}$, and 
TABLE 1: Physicochemical and microbiological characteristics of R.B.N.S. sugar mill effluent.

\begin{tabular}{|c|c|c|c|c|c|c|c|}
\hline \multirow{2}{*}{ Parameter } & \multirow{2}{*}{$0(\mathrm{BWW})^{\mathrm{a}}$} & \multicolumn{5}{|c|}{ Sugar mill effluent concentration (\%) } & \multirow{2}{*}{${ }^{b}$ BIS for irrigation wate } \\
\hline & & 20 & 40 & 60 & 80 & 100 & \\
\hline TDS $\left(\mathrm{mg} \mathrm{L}^{-1}\right)$ & 198.50 & $1456.00^{* *}$ & $2948.00^{* *}$ & $4368.00^{* *}$ & $5676.00^{* *}$ & $6182.00^{* *}$ & 1900 \\
\hline Turbidity (NTU) & 4.46 & $13.68^{*}$ & $18.88^{* *}$ & $24.67^{* *}$ & $30.98^{* *}$ & $34.67^{* *}$ & 10 \\
\hline $\mathrm{EC}\left(\mathrm{dS} \mathrm{cm}^{-1}\right)$ & 0.34 & $2.68^{*}$ & $4.76^{*}$ & $6.89^{* *}$ & $8.96^{* *}$ & $9.85^{* *}$ & $-^{\mathrm{c}}$ \\
\hline $\mathrm{pH}$ & 7.50 & $7.78 \mathrm{~ns}$ & $7.87 \mathrm{~ns}$ & $7.98 \mathrm{~ns}$ & $8.82^{*}$ & $8.98^{*}$ & $5.5-9.0$ \\
\hline $\mathrm{DO}\left(\mathrm{mg} \mathrm{L}^{-1}\right)$ & 8.24 & $4.89^{*}$ & $3.78^{*}$ & $2.56^{* *}$ & $2.34^{* *}$ & $1.23^{* *}$ & - \\
\hline $\mathrm{BOD}\left(\mathrm{mg} \mathrm{L}^{-1}\right)$ & 3.83 & $184.36^{* *}$ & $368.89^{* *}$ & $779.68^{* *}$ & $1238.96^{* *}$ & $1637.58^{* *}$ & 100 \\
\hline $\operatorname{COD}\left(\mathrm{mg} \mathrm{L}^{-1}\right)$ & 5.88 & $235.68^{* *}$ & $576.45^{* *}$ & $1138.78^{* *}$ & $1699.78^{* *}$ & $2267.96^{* *}$ & 250 \\
\hline $\mathrm{CL}^{-}\left(\mathrm{mg} \mathrm{L}^{-1}\right)$ & 15.68 & $148.52^{* *}$ & $339.84^{* *}$ & $658.47^{* *}$ & $947.58^{* *}$ & $1249.82^{* *}$ & 500 \\
\hline $\mathrm{HCO}_{3}{ }^{-}\left(\mathrm{mg} \mathrm{L}^{-1}\right)$ & 282.00 & $313.15^{*}$ & $337.14^{*}$ & $379.48^{*}$ & $518.23^{* *}$ & $669.86^{* *}$ & - \\
\hline $\mathrm{CO}_{3}^{-2}\left(\mathrm{mg} \mathrm{L}^{-1}\right)$ & 105.75 & $137.47^{*}$ & $178.85^{*}$ & $199.69^{*}$ & $216.36^{* *}$ & $249.59^{* *}$ & - \\
\hline $\mathrm{Na}^{+}\left(\mathrm{mg} \mathrm{L}^{-1}\right)$ & 9.65 & $35.36^{* *}$ & $84.25^{* *}$ & $148.71^{* *}$ & $219.58^{* *}$ & $288.96^{* *}$ & - \\
\hline $\mathrm{K}^{+}\left(\mathrm{mg} \mathrm{L}^{-1}\right)$ & 5.54 & $46.58^{* *}$ & $98.67^{* *}$ & $189.47^{* *}$ & $278.37^{* *}$ & $359.82^{* *}$ & - \\
\hline $\mathrm{Ca}^{2+}\left(\mathrm{mg} \mathrm{L}^{-1}\right)$ & 23.46 & $113.26^{*}$ & $258.93^{*}$ & $454.45^{* *}$ & $662.39^{* *}$ & $852.79^{* *}$ & 200 \\
\hline $\mathrm{Mg}^{2+}\left(\mathrm{mg} \mathrm{L}^{-1}\right)$ & 12.15 & $34.64^{*}$ & $69.46^{*}$ & $112.58^{* *}$ & $158.96^{* *}$ & $197.95^{* *}$ & - \\
\hline TKN $\left(\mathrm{mg} \mathrm{L}^{-1}\right)$ & 24.27 & $44.54^{*}$ & $65.88^{*}$ & $96.37^{* *}$ & $118.24^{* *}$ & $149.78^{* *}$ & 100 \\
\hline $\mathrm{NO}_{3}{ }^{2-}\left(\mathrm{mg} \mathrm{L}^{-1}\right)$ & 25.17 & $104.74^{*}$ & $236.55^{*}$ & $422.87^{* *}$ & $495.64^{* *}$ & $788.88^{* *}$ & 100 \\
\hline $\mathrm{PO}_{4}{ }^{3-}\left(\mathrm{mg} \mathrm{L}^{-1}\right)$ & 0.04 & $32.44^{* *}$ & $73.69^{* *}$ & $146.57^{* *}$ & $219.78^{* *}$ & $296.89^{* *}$ & - \\
\hline $\mathrm{SO}_{4}{ }^{2-}\left(\mathrm{mg} \mathrm{L}^{-1}\right)$ & 17.64 & $114.12^{*}$ & $252.36^{*}$ & $464.85^{* *}$ & $684.36^{* *}$ & $892.77^{* *}$ & 1000 \\
\hline $\mathrm{Fe}^{3+}\left(\mathrm{mg} \mathrm{L}^{-1}\right)$ & 0.28 & $7.36^{* *}$ & $14.86^{* *}$ & $21.48^{* *}$ & $28.44^{* *}$ & $34.68^{* *}$ & 1.0 \\
\hline $\mathrm{Zn}^{2+}\left(\mathrm{mg} \mathrm{L}^{-1}\right)$ & 0.06 & $4.79^{* *}$ & $9.84^{* *}$ & $13.78^{* *}$ & $18.98^{* *}$ & $22.36^{* *}$ & 15 \\
\hline $\mathrm{Cd}^{2+}\left(\mathrm{mg} \mathrm{L}^{-1}\right)$ & 0.01 & $1.86^{* *}$ & $3.12^{* *}$ & $5.24^{* *}$ & 6.48 & $8.36^{* *}$ & 2.00 \\
\hline $\mathrm{Cu}^{2+}\left(\mathrm{mg} \mathrm{L}^{-1}\right)$ & 0.04 & $1.55^{* *}$ & $2.98^{* *}$ & $4.99^{* *}$ & $6.78^{* *}$ & $8.96^{* *}$ & 3.00 \\
\hline $\mathrm{Mn}^{2+}\left(\mathrm{mg} \mathrm{L}^{-1}\right)$ & 0.02 & $4.45^{* *}$ & $7.98^{* *}$ & $9.25^{* *}$ & $11.22^{* *}$ & $16.48^{* *}$ & 1.00 \\
\hline $\mathrm{Cr}^{3+}\left(\mathrm{mg} \mathrm{L}^{-1}\right)$ & 0.01 & $0.28^{* *}$ & $0.59^{* *}$ & $1.26^{* *}$ & $1.45^{* *}$ & $1.67^{* *}$ & 2.00 \\
\hline $\operatorname{SPC}\left(\mathrm{SPC} \mathrm{mL} \mathrm{m}^{-1}\right)$ & $4.8 \times 10^{3}$ & $5.79 \times 10^{6 * *}$ & $4.98 \times 10^{8 * *}$ & $7.96 \times 10^{10 * *}$ & $6.97 \times 10^{12 * *}$ & $8.78 \times 10^{14 * *}$ & 10000 \\
\hline Total coliforms $\left(\mathrm{MPN1} 100 \mathrm{~mL}^{-1}\right)$ & $2.4 \times 10^{2}$ & $4.48 \times 10^{4 * *}$ & $5.79 \times 10^{6 * *}$ & $6.87 \times 10^{8 * *}$ & $7.78 \times 10^{10 * *}$ & $6.99 \times 10^{12 * *}$ & 5000 \\
\hline
\end{tabular}

ns, ${ }^{*},{ }^{* *}$ : nonsignificant or significant at $P \leq 0.05$ or $P \leq 0.01$, respectively, least squares means.

${ }^{a}$ BWW: well water control.

bIS: Bureau of Indian standard.

c“-": not given in standard.

TABLE 2: ANOVA for the effect of sugar mill effluent on soil characteristics.

\begin{tabular}{|c|c|c|c|c|c|c|c|c|c|c|c|c|c|c|c|c|c|c|}
\hline Source & WHC & $\mathrm{BD}$ & $\mathrm{EC}$ & $\mathrm{pH}$ & OC & $\mathrm{Na}^{+}$ & $\mathrm{K}^{+}$ & $\mathrm{Ca}^{2+}$ & $\mathrm{Mg}^{2+}$ & $\mathrm{Fe}^{3+}$ & TKN & $\mathrm{PO}_{4}{ }^{3-}$ & $\mathrm{SO}_{4}^{-2}$ & $\mathrm{Zn}^{2+}$ & $\mathrm{Cd}^{2+}$ & $\mathrm{Cu}^{2+}$ & $\mathrm{Mn}^{2+}$ & $\mathrm{Cr}^{3+}$ \\
\hline Season (S) & ns & ns & ns & ns & $*$ & * & $*$ & $*$ & $*$ & $*$ & $*$ & $*$ & * & $*$ & * & * & ns & $*$ \\
\hline SME concentration $(\mathrm{C})$ & ns & ns & $* *$ & $*$ & $* *$ & $* *$ & $*$ & $*$ & * & $* *$ & $* *$ & $* *$ & $* *$ & $* *$ & $* *$ & $* *$ & * & $* *$ \\
\hline Interaction $\mathrm{S} \times \mathrm{C}$ & ns & ns & $*$ & $*$ & $* *$ & $* *$ & $* *$ & $* *$ & $* *$ & $* *$ & $* *$ & $* *$ & $* *$ & $* *$ & $* *$ & $* *$ & $* *$ & $* *$ \\
\hline
\end{tabular}

ns, ${ }^{*},{ }^{* *}$ : nonsignificant or significant at $P \leq 0.05$ or $P \leq 0.01$, ANOVA; SME: sugar mill effluent.

$\mathrm{Cr}^{3+}$ in the soil (Table 2). The values of $\mathrm{Zn}^{2+}, \mathrm{Cd}^{2+}, \mathrm{Cu}^{2+}$, $\mathrm{Mn}^{2+}$, and $\mathrm{Cr}^{3+}$ in the soil were increased when the effluent concentrations increased (Table 4). The concentration of Mn was highest while that of $\mathrm{Cr}$ was lowest after sugar mill effluent irrigation in both seasons. The contamination factor (Cf) of the heavy metals indicated the contamination after sugar mill effluent irrigation. The Cf of heavy metals in the soil was in the order of $\mathrm{Mn}^{2+}>\mathrm{Zn}^{2+}>\mathrm{Cu}^{2+}>\mathrm{Cd}^{2+}>$ $\mathrm{Cr}^{3+}$ after irrigation with sugar mill effluent in both seasons (Figure 1). Thus, fertigation increased the nutrients as well as metals in the soil.

\subsection{Effects of Sugar Mill Effluent Fertigation on Z. mays}

3.3.1. Effects on Germination. Seed germination of $Z$. mays was affected by sugar mill effluent treatment (Table 6, Figure 2). At 0-15 days after sowing, the best germination (96.00\% and $94.00 \%)$ was noted with $40 \%$ sugar mill effluent and the least $(86.00 \%$ and $85.00 \%)$ was recorded with $100 \%$ sugar mill effluent. Germination of $Z$. mays was observed to be negatively correlated with sugar mill effluent concentrations in both seasons (Table 9). The ANOVA indicated that the seasons showed insignificant $(P>0.05)$ effect on seed 
TABLE 3: Effects of sugar mill effluent concentration and season interaction on physicochemical characteristics of soil used in the cultivation of $Z$. mays in both seasons.

\begin{tabular}{|c|c|c|c|c|c|c|c|}
\hline Season $\times \%$ SME & $\mathrm{EC}\left(\mathrm{dS} \cdot \mathrm{m}^{-1}\right)$ & $\mathrm{pH}$ & $\mathrm{OC}\left(\mathrm{mg} \cdot \mathrm{kg}^{-1}\right)$ & $\mathrm{Na}^{+}\left(\mathrm{mg} \cdot \mathrm{kg}^{-1}\right)$ & $\mathrm{K}^{+}\left(\mathrm{mg} \cdot \mathrm{kg}^{-1}\right)$ & $\mathrm{Ca}^{2+}\left(\mathrm{mg} \cdot \mathrm{kg}^{-1}\right)$ & $\mathrm{Mg}^{2+}\left(\mathrm{mg} \cdot \mathrm{kg}^{-1}\right)$ \\
\hline \multicolumn{8}{|l|}{ Rainy } \\
\hline 0 & 1.92 & 7.63 & 0.52 & 25.48 & 164.53 & 16.52 & 23.43 \\
\hline 20 & $2.98 \mathrm{~ns}$ & $7.82 \mathrm{~ns}$ & $3.68^{*}$ & $33.96^{*}$ & $188.55 \mathrm{~ns}$ & $29.67^{*}$ & $38.86^{*}$ \\
\hline 40 & $5.66^{*}$ & $8.01 \mathrm{~ns}$ & $6.34^{* *}$ & $60.85^{*}$ & $237.94^{* *}$ & $69.88^{* *}$ & $59.96^{* *}$ \\
\hline 60 & $7.78^{*}$ & $8.26^{*}$ & $10.92^{* *}$ & $77.86^{*}$ & $265.68^{* *}$ & $98.65^{* *}$ & $7.36^{* *}$ \\
\hline 80 & $9.86^{*}$ & $8.43^{*}$ & $15.88^{* *}$ & $87.63^{* *}$ & $290.98^{*}$ & $128.52^{* *}$ & $74.82^{*}$ \\
\hline 100 & $12.88^{* *}$ & $8.63^{*}$ & $18.86^{* *}$ & $96.76^{* *}$ & $324.75^{*}$ & $144.87^{* *}$ & $88.69^{*}$ \\
\hline \multicolumn{8}{|l|}{ Summer } \\
\hline 0 & 1.94 & 7.64 & 0.54 & 25.68 & 165.88 & 17.86 & 23.69 \\
\hline 20 & $3.72 \mathrm{~ns}$ & $7.94 \mathrm{~ns}$ & $4.01^{*}$ & $35.69^{*}$ & $192.86 \mathrm{~ns}$ & $31.75^{*}$ & $48.85^{*}$ \\
\hline 40 & $6.36^{*}$ & $8.15 \mathrm{~ns}$ & $6.96^{* *}$ & $65.25^{*}$ & $248.68^{* *}$ & $79.84^{* *}$ & $72.32^{* *}$ \\
\hline 60 & $8.48^{*}$ & $8.37^{*}$ & $17.86^{* *}$ & $87.36^{*}$ & $278.98^{*}$ & $117.56^{* *}$ & $78.69^{* *}$ \\
\hline 80 & $11.55^{*}$ & $8.52^{*}$ & $19.78^{* *}$ & $89.69^{* *}$ & $299.36^{*}$ & $148.69^{* *}$ & $85.55^{*}$ \\
\hline 100 & $14.96^{* *}$ & $8.72^{*}$ & $21.84^{* *}$ & $99.36^{* *}$ & $334.88^{*}$ & $159.96^{* *}$ & $98.69^{*}$ \\
\hline
\end{tabular}

ns, ${ }^{*}{ }^{* *}$ : nonsignificant or significant at $P \leq 0.05$ or $P \leq 0.01$, respectively, least squares means; SME: sugar mill effluent.

TABLE 4: Effects of sugar mill effluent concentration and season interaction on physicochemical characteristics of soil used in the cultivation of $Z$. mays in both seasons.

\begin{tabular}{|c|c|c|c|c|c|c|c|c|c|}
\hline Season $\times \% \mathrm{SME}$ & $\begin{array}{c}\mathrm{TKN} \\
\left(\mathrm{mg} \mathrm{kg}^{-1}\right)\end{array}$ & $\begin{array}{c}\mathrm{PO}_{4}{ }^{3-} \\
\left(\mathrm{mg} \mathrm{kg}^{-1}\right)\end{array}$ & $\begin{array}{c}\mathrm{SO}_{4}{ }^{2-} \\
\left(\mathrm{mg} \mathrm{kg}^{-1}\right)\end{array}$ & $\begin{array}{c}\mathrm{Fe}^{3+} \\
\left(\mathrm{mg} \mathrm{kg}^{-1}\right)\end{array}$ & $\begin{array}{c}\mathrm{Zn}^{2+} \\
\left(\mathrm{mg} \mathrm{kg}^{-1}\right)\end{array}$ & $\begin{array}{c}\mathrm{Cd}^{2+} \\
\left(\mathrm{mg} \mathrm{kg}^{-1}\right)\end{array}$ & $\begin{array}{c}\mathrm{Cu}^{2+} \\
\left(\mathrm{mg} \mathrm{kg}^{-1}\right)\end{array}$ & $\begin{array}{c}\mathrm{Mn}^{2+} \\
\left(\mathrm{mg} \mathrm{kg}^{-1}\right)\end{array}$ & $\begin{array}{c}\mathrm{Cr}^{3+} \\
\left(\mathrm{mg} \mathrm{kg}^{-1}\right)\end{array}$ \\
\hline \multicolumn{10}{|l|}{ Rainy } \\
\hline 0 & 36.88 & 63.69 & 84.58 & 4.58 & 0.56 & 0.33 & 1.14 & 0.59 & 0.29 \\
\hline 20 & $82.36^{* *}$ & $98.47^{*}$ & $131.36^{*}$ & $8.86 \mathrm{~ns}$ & $2.96 \mathrm{~ns}$ & $1.22 \mathrm{~ns}$ & $4.40 \mathrm{~ns}$ & $3.05 \mathrm{~ns}$ & $0.29 \mathrm{~ns}$ \\
\hline 40 & $174.33^{* *}$ & $145.64^{*}$ & $156.78^{* *}$ & $19.96^{*}$ & $4.30^{*}$ & $1.56^{*}$ & $6.68^{*}$ & $6.09 \mathrm{~ns}$ & $0.74^{*}$ \\
\hline 60 & $295.29^{* *}$ & $176.93^{* *}$ & $186.68^{* *}$ & $38.78^{*}$ & $6.87^{*}$ & $1.94^{*}$ & $9.36^{* *}$ & $8.15 \mathrm{~ns}$ & $0.99^{*}$ \\
\hline 80 & $358.75^{* *}$ & $183.3^{* *}$ & $254.36^{* *}$ & $58.47^{* *}$ & $8.96^{* *}$ & $2.11^{* *}$ & $12.36^{* *}$ & $10.25^{* *}$ & $1.58^{*}$ \\
\hline 100 & $438.64^{*}$ & $248.75^{* *}$ & $287.96^{* *}$ & $75.68^{* *}$ & $12.68^{* *}$ & $3.66^{* *}$ & $15.48^{* *}$ & $13.69^{*}$ & $1.96^{* *}$ \\
\hline \multicolumn{10}{|l|}{ Summer } \\
\hline 0 & 37.45 & 64.24 & 84.88 & 4.60 & 0.57 & 0.34 & 1.16 & 0.62 & 0.32 \\
\hline 20 & $86.49^{* *}$ & $96.79^{*}$ & $139.69^{*}$ & $9.36 \mathrm{~ns}$ & $3.29 \mathrm{~ns}$ & $1.34 \mathrm{~ns}$ & $4.76 \mathrm{~ns}$ & $3.36 \mathrm{~ns}$ & $0.45 \mathrm{~ns}$ \\
\hline 40 & $182.88^{* *}$ & $143.85^{*}$ & $177.84^{* *}$ & $23.25^{*}$ & $4.64^{*}$ & $1.78^{*}$ & $7.81^{*}$ & $6.84 \mathrm{~ns}$ & $0.84^{*}$ \\
\hline 60 & $324.24^{* *}$ & $184.25^{* *}$ & $196.55^{* *}$ & $45.63^{*}$ & $7.49^{*}$ & $2.06^{*}$ & $10.15^{* *}$ & $9.63 \mathrm{~ns}$ & $1.86^{*}$ \\
\hline 80 & $398.96^{* *}$ & $193.8^{* *}$ & $268.41^{* *}$ & $67.75^{* *}$ & $9.76^{* *}$ & $2.36^{*}$ & $13.48^{* *}$ & $12.44^{* *}$ & $1.96^{*}$ \\
\hline 100 & $460.88^{* *}$ & $258.69^{* *}$ & $294.68^{* *}$ & $78.87^{* *}$ & $14.12^{* *}$ & $4.25^{*}$ & $16.78^{* *}$ & $15.56^{*}$ & $2.36^{* *}$ \\
\hline
\end{tabular}

$\mathrm{ns},{ }^{*},{ }^{* *}$ : nonsignificant or significant at $P \leq 0.05$ or $P \leq 0.01$, respectively, least squares means analysis; SME: sugar mill effluent.

germination and relative toxicity. Sugar mill effluent concentrations and their interaction with seasons affected seed germination of $Z$. mays, but not relative toxicity (Table 6).

The maximum RT (106.25\% and $104.78 \%)$ of sugar mill effluent against germination of $Z$. mays was observed with $100 \%$ sugar mill effluent and it was positively correlated with all concentrations of sugar mill effluent in both seasons (Table 9, Figure 3). The findings were very much in accordance with Radhouane [34], who reported that the germination of millet cultivars was decreased when concentration of waste effluent increased from $0 \%$ to $100 \%$.

In the present investigation, the higher concentration of sugar mill effluent did not support seed germination. The higher concentration of sugar mill effluent lowered the seed germination of $Z$. mays, and it is likely due to the presence of higher salt content in the effluent at these concentrations. The excessive quantities of inorganic salts and the higher EC, since these biotoxic substances present in the effluent at higher concentrations, increase the salinity and osmotic pressure, which might have inhibited seed germination by altering the interaction of seed and water which is necessary for triggering enzyme activity as also reported by findings of earlier researchers $[26,34,48,49]$.

3.3.2. Effects on Vegetative Growth Stage. Vegetative growth of $Z$. mays at 45 days was affected in both seasons (Table 6). The minimum root length $(12.15$ and $13.21 \mathrm{~cm})$, number of leaves (10.00 and 12.00), and dry weight/plant (55.87 and 
TABLE 5: Coefficient of correlation $(r)$ between sugar mill effluent and soil characteristics in both seasons.

\begin{tabular}{|c|c|c|}
\hline Effluent/soil characteristics & Season & $r$-value \\
\hline \multirow{2}{*}{ Sugar mill effluent versus soil WHC } & Rainy & -0.97 \\
\hline & Summer & -0.97 \\
\hline \multirow{2}{*}{ Sugar mill effluent versus soil BD } & Rainy & -0.96 \\
\hline & Summer & -0.96 \\
\hline \multirow{2}{*}{ Sugar mill effluent versus soil EC } & Rainy & +0.98 \\
\hline & Summer & +0.98 \\
\hline \multirow{2}{*}{ Sugar mill effluent versus soil $\mathrm{pH}$} & Rainy & -0.96 \\
\hline & Summer & -0.96 \\
\hline \multirow{2}{*}{ Sugar mill effluent versus soil OC } & Rainy & +0.99 \\
\hline & Summer & +0.99 \\
\hline \multirow{2}{*}{ Sugar mill effluent versus soil $\mathrm{Na}^{+}$} & Rainy & +0.97 \\
\hline & Summer & +0.97 \\
\hline \multirow{2}{*}{ Sugar mill effluent versus soil $\mathrm{K}^{+}$} & Rainy & +0.87 \\
\hline & Summer & +0.87 \\
\hline \multirow{2}{*}{ Sugar mill effluent versus soil $\mathrm{Ca}^{2+}$} & Rainy & +0.76 \\
\hline & Summer & +0.76 \\
\hline \multirow{2}{*}{ Sugar mill effluent versus soil $\mathrm{Mg}^{2+}$} & Rainy & +0.63 \\
\hline & Summer & +0.63 \\
\hline \multirow{2}{*}{ Sugar mill effluent versus soil TKN } & Rainy & +0.99 \\
\hline & Summer & +0.99 \\
\hline \multirow{2}{*}{ Sugar mill effluent versus soil $\mathrm{PO}_{4}^{3-}$} & Rainy & +0.99 \\
\hline & Summer & +0.99 \\
\hline \multirow{2}{*}{ Sugar mill effluent versus soil $\mathrm{SO}_{4}{ }^{2-}$} & Rainy & +0.96 \\
\hline & Summer & +0.96 \\
\hline \multirow{2}{*}{ Sugar mill effluent versus soil $\mathrm{Fe}^{3+}$} & Rainy & +0.99 \\
\hline & Summer & +0.99 \\
\hline \multirow{2}{*}{ Sugar mill effluent versus soil $\mathrm{Zn}^{2+}$} & Rainy & +0.94 \\
\hline & Summer & +0.94 \\
\hline \multirow{2}{*}{ Sugar mill effluent versus soil $\mathrm{Cd}^{2+}$} & Rainy & +0.97 \\
\hline & Summer & +0.97 \\
\hline \multirow{2}{*}{ Sugar mill effluent versus soil $\mathrm{Cu}^{2+}$} & Rainy & +0.99 \\
\hline & Summer & +0.99 \\
\hline \multirow{2}{*}{ Sugar mill effluent versus soil $\mathrm{Mn}^{2+}$} & Rainy & +0.99 \\
\hline & Summer & +0.99 \\
\hline \multirow{2}{*}{ Sugar mill effluent versus soil $\mathrm{Cr}^{3+}$} & Rainy & +0.99 \\
\hline & Summer & +0.99 \\
\hline
\end{tabular}

$57.98 \mathrm{~g})$ of $Z$. mays were recorded with control while moderate root length $(12.44$ and $13.63 \mathrm{~cm})$, number of leaves $(12.00$ and 13.00), and dry weight/plant (44.78 and $45.96 \mathrm{~g}$ ) of $Z$. mays were observed with $100 \%$ sugar mill effluent in both seasons. The maximum root length (14.24 and $16.12 \mathrm{~cm})$, number of leaves (14.00 and 16.00), and dry weight/plant (65.47 and $68.68 \mathrm{~g}$ ) of $Z$. mays were noted with $40 \%$ concentration of sugar mill effluent in both seasons. Sugar mill effluent concentrations, the seasons, and their interaction had no effect on root length, number of leaves, and dry weight of the Z. mays (Table 6).

Maximum plant height, fresh weight, chlorophyll content, and LAI/plant of $Z$. mays were due to the treatment with the $40 \%$ concentration of sugar mill effluent in both

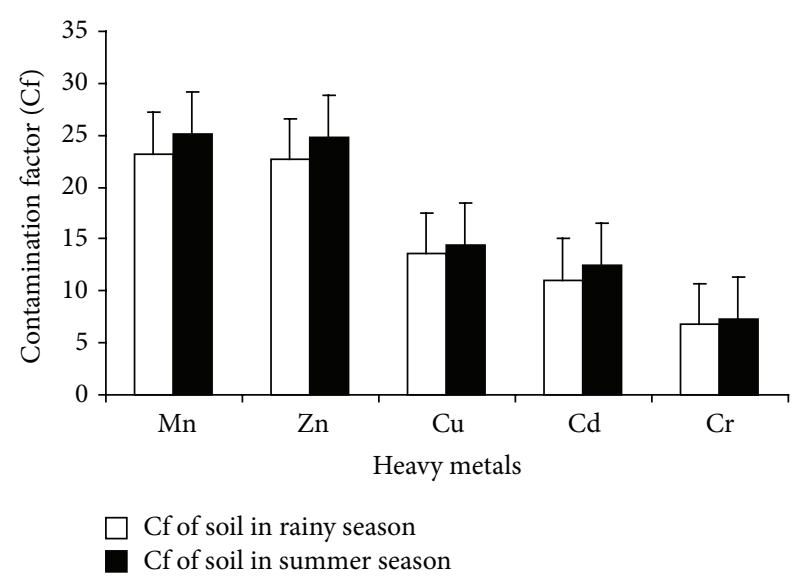

Figure 1: Contamination factor of heavy metals in soil after irrigation with sugar mill effluent. Error bars are standard error of the mean.

seasons (Table 7). The ANOVA indicated that sugar mill effluent concentrations affected plant height, fresh weight, chlorophyll content, and LAI/plant of Z. mays (Tables 6 and 7). The seasons had no effect on plant height, fresh weight, chlorophyll content, and LAI/plant of $Z$. mays. The interaction of seasons and sugar mill effluent concentrations affected plant height, fresh weight, chlorophyll content, and LAI/plant of Z. mays (Tables 6 and 7).

Plant height, number of leaves, fresh weight, dry weight, chlorophyll content, and LAI/plant of Z. mays were noted to be positively correlated with sugar mill effluent concentrations in both seasons (Table 9). Root length was observed to be positively correlated with sugar mill effluent concentrations in the rainy season while it was noted to be negatively correlated in summer season (Table 9). Osaigbovo and Orhue [11] reported the maximum plant height, number of leaves, and chlorophyll content at $25 \%$ of pharmaceutical effluent treatment on maize plant. Khan et al. [10] reported that treated effluent irrigation increases chlorophyll and protein contents in pearl millet plants (P. glaucum L.) at the 25 and $50 \%$ sugar mill effluent concentrations followed by a decrease in $75 \%$ and $100 \%$ sugar mill effluent.

Vegetative growth of $Z$. mays was lowered at higher concentrations of sugar mill effluent. A high EC indicates higher salt content in the higher sugar mill effluent concentrations, which lowered the plant height, root length, number of leaves, fresh weight, dry weight, chlorophyll content, and $\mathrm{LAI} /$ plant of $Z$. mays. Vegetative growth is associated with the development of new shoots, twigs, leaves, and leaf area. Plant height, fresh weight, chlorophyll content, and LAI/plant of $Z$. mays were recorded higher at $40 \%$ of sugar mill effluent; it may be due to the maximum uptake of nitrogen, phosphorus, and potassium by plants. The improvement of vegetative growth may be attributed to the role of potassium in nutrient and sugar translocation in plants and turgor pressure in plant cells [50]. It is also involved in cell enlargement and in triggering young tissue or meristematic growth [51]. Chlorophyll content was noted higher due to the use of $40 \%$ sugar mill effluent in both seasons and is likely due to $\mathrm{Fe}^{3+}$, 
TABLE 6: ANOVA for the effect of sugar mill effluent on agronomical characteristics of Z. mays.

\begin{tabular}{|c|c|c|c|c|c|c|c|c|c|c|c|c|}
\hline Source & $\begin{array}{c}\text { Seed } \\
\text { germination }\end{array}$ & $\begin{array}{l}\text { Relative } \\
\text { toxicity }\end{array}$ & $\begin{array}{l}\text { Plant } \\
\text { height }\end{array}$ & $\begin{array}{l}\text { Root } \\
\text { length }\end{array}$ & $\begin{array}{c}\text { Number } \\
\text { of } \\
\text { leaves }\end{array}$ & $\begin{array}{l}\text { Fresh } \\
\text { weight }\end{array}$ & $\begin{array}{c}\text { Dry } \\
\text { weight }\end{array}$ & $\begin{array}{l}\text { Chlorophyll } \\
\text { content }\end{array}$ & LAI & $\begin{array}{l}\text { Cob } \\
\text { length }\end{array}$ & CY/plant & $\mathrm{HI}$ \\
\hline Season (S) & ns & ns & ns & ns & ns & ns & ns & ns & ns & * & * & $*$ \\
\hline $\begin{array}{l}\text { SME concentration } \\
\text { (C) }\end{array}$ & $*$ & ns & $*$ & ns & ns & * & ns & $*$ & $*$ & $*$ & $*$ & * \\
\hline Interaction $\mathrm{S} \times \mathrm{C}$ & * & $\mathrm{ns}$ & * & $\mathrm{ns}$ & $\mathrm{ns}$ & * & $\mathrm{ns}$ & * & * & * & * & * \\
\hline
\end{tabular}

ns, ${ }^{*}$ : nonsignificant or significant at $P \leq 0.05$, ANOVA; SME: sugar mill effluent.

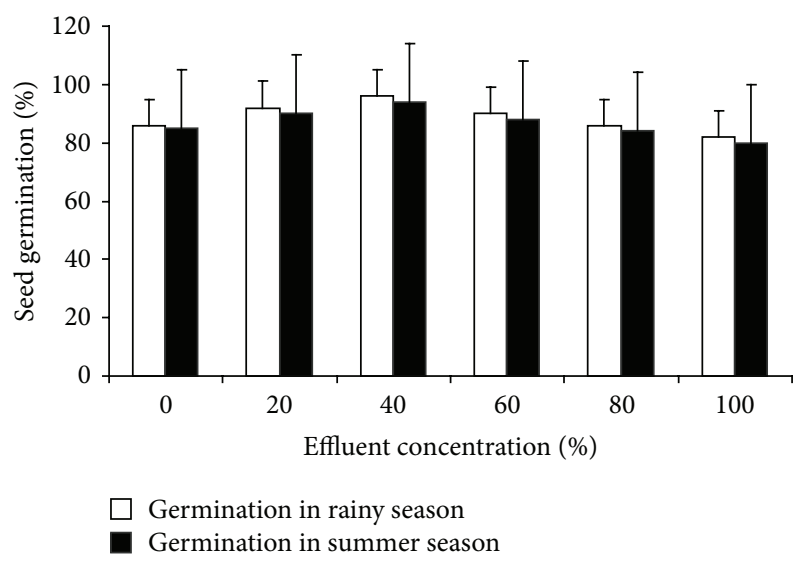

FIGURE 2: Seed germination of $Z$. mays after irrigation with sugar mill effluent. Error bars are standard error of the mean.

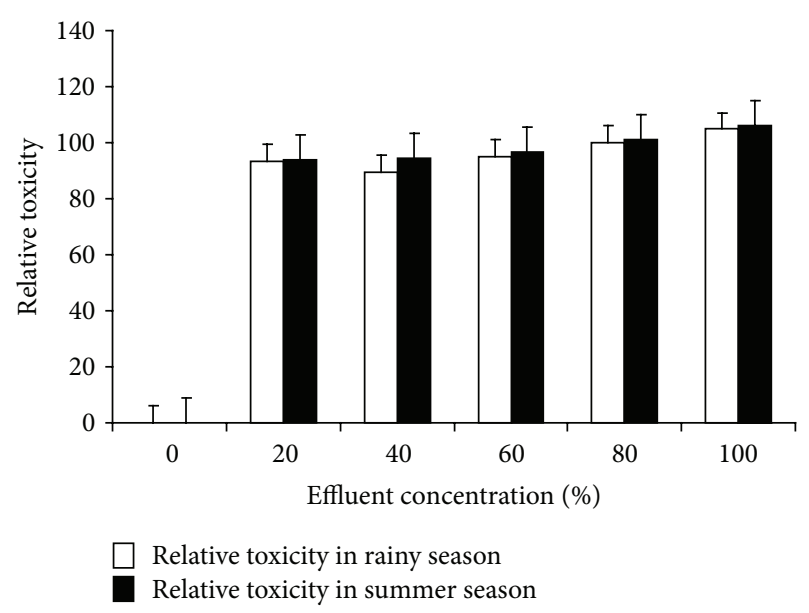

FIgURE 3: Relative toxicity of sugar mill effluent against seed germination of $Z$. mays. Error bars are standard error of the mean.

$\mathrm{Mg}^{2+}$, and $\mathrm{Mn}^{2+}$ contents in the sugar mill effluent, which are associated with chlorophyll synthesis (Porra [36]). Thus, 40\% sugar mill effluent concentration contains optimum contents of nutrients required for maximum vegetative growth of $Z$. mays.

3.3.3. Effects on Maturity Stage. At maturity stage (90 days after sowing) the cob length and crop yield/plant of $Z$. mays were noted with $40 \%$ sugar mill effluent in both seasons. Cob length and crop yield of $Z$. mays were decreased as the sugar mill effluent concentration increased (Table 7). Maximum HI (156.04 and $157.76 \%$ ) of $Z$. mays was recorded with $40 \%$ concentration of sugar mill effluent while minimum $\mathrm{HI}$ (134.58\% and $138.92 \%)$ was observed with $100 \%$ sugar mill effluent in both seasons. Sugar mill effluent concentrations affected cob length and crop yield of $Z$. mays but not seasons; interaction of seasons and sugar mill effluent concentrations had an effect on cob length and crop yield of $Z$. mays (Table 6). The seasons and sugar mill effluent concentrations had no effect on harvest index (HI) of $Z$. mays. Crop yield of $Z$. mays was observed to be positively correlated with sugar mill effluent concentrations in the rainy season while it was noted to be negatively correlated in the summer season (Table 9). The results were supported by Zalawadia and Raman [52], who observed that crop yield of sorghum was decreased with increase in distillery effluent concentrations.

Nitrogen $(\mathrm{N})$ and phosphorus $(\mathrm{P})$ are essential for flowering and grains filling. More quantity of $\mathrm{N}$ can delay or prevent flowering while $\mathrm{P}$ deficiency is sometimes associated with poor flower production or flower abortion. Maximum cob length of $Z$. mays was with the $40 \%$ sugar mill effluent; it might be due to the fact that this concentration contains sufficient $\mathrm{N}$ and P. Furthermore, $\mathrm{N}$ and $\mathrm{P}$ prevent flower abortion so grains filling occurs [53]. Cob length of $Z$. mays was decreased at higher concentrations of sugar mill effluent. This is likely due to the higher content of metals in the soil, which inhibits uptake of $\mathrm{PO}_{4}{ }^{3-}$ and $\mathrm{K}^{+}$by plants at higher concentrations [54]. The role of $\mathrm{K}^{+}, \mathrm{Fe}^{3+}, \mathrm{Mg}^{2+}$, and $\mathrm{Mn}^{2+}$ at maturity is important and associated with synthesis of chlorophyll and enhances the formation of grains at harvest $[53,55]$. The $\mathrm{K}^{+}, \mathrm{Fe}^{3+}, \mathrm{Mg}^{2+}$ and $\mathrm{Mn}^{2+}$ contents could benefit cob formation, grains filling, and yield of maize (Z. mays L.) as reported by Ezhilvannan et al. [4]. The $40 \%$ concentration of sugar mill effluent favored the grains formation and crop yield of $Z$. mays. This is likely due to the presence of $\mathrm{K}^{+}, \mathrm{Fe}^{3+}, \mathrm{Mg}^{2+}$, and $\mathrm{Mn}^{2+}$ contents in $40 \%$ sugar mill effluent, while higher sugar mill effluent concentrations lowered grains formation and crop yield of $Z$. mays.

\subsection{Effects on Biochemical Constituents and Micronutrients in} $Z$. mays. Seasons, sugar mill effluent concentrations, and the interaction of seasons and sugar mill effluent concentrations affected all the metals like crude proteins, crude fiber, total carbohydrates, $\mathrm{Zn}^{2+}, \mathrm{Cd}^{2+}, \mathrm{Cu}^{2+}, \mathrm{Mn}^{2+}$, and $\mathrm{Cr}^{3+}$ in $Z$. mays (Table 8). Maximum crude proteins, crude fiber, and total 
TABLE 7: Effects of sugar mill concentration and season interaction on agronomical parameters of Z. mays.

\begin{tabular}{|c|c|c|c|c|c|c|}
\hline Season $\times \%$ SME & $\begin{array}{l}\text { Plant height } \\
(\mathrm{cm})\end{array}$ & $\begin{array}{c}\text { Fresh } \\
\text { weight }(\mathrm{g})\end{array}$ & $\begin{array}{l}\text { Chlorophyll content } \\
\text { (mg/g.f.wt.) }\end{array}$ & LAI & $\begin{array}{l}\text { Cob length } \\
\text { (cm) }\end{array}$ & $\begin{array}{l}\text { Crop yield/plant } \\
\text { (g) }\end{array}$ \\
\hline \multicolumn{7}{|l|}{ Rainy } \\
\hline 0 & 176.86 & 123.13 & 3.32 & 2.35 & 16.58 & 54.42 \\
\hline 20 & $184.56^{*}$ & $143.56^{*}$ & $3.95 \mathrm{~ns}$ & $3.55 \mathrm{~ns}$ & $18.63 \mathrm{~ns}$ & $68.75 \mathrm{~ns}$ \\
\hline 40 & $204.58^{*}$ & $165.34^{*}$ & $4.81^{*}$ & $5.19^{*}$ & $22.36^{*}$ & $80.59^{*}$ \\
\hline 60 & $192.85^{*}$ & $152.98^{*}$ & $4.71^{*}$ & $4.76^{*}$ & $20.36^{*}$ & $75.32^{*}$ \\
\hline 80 & $188.37^{*}$ & $142.34^{*}$ & $4.42^{*}$ & $4.43^{*}$ & $19.48^{*}$ & $69.67 \mathrm{~ns}$ \\
\hline 100 & $180.59^{*}$ & $130.56 \mathrm{~ns}$ & $3.45 \mathrm{~ns}$ & $3.81 \mathrm{~ns}$ & $18.18 \mathrm{~ns}$ & $62.88 \mathrm{~ns}$ \\
\hline \multicolumn{7}{|l|}{ Summer } \\
\hline 0 & 170.69 & 115.34 & 3.12 & 2.12 & 14.46 & 50.74 \\
\hline 20 & $180.25^{*}$ & $137.56^{*}$ & $3.46 \mathrm{~ns}$ & $3.46 \mathrm{~ns}$ & $16.63 \mathrm{~ns}$ & $62.36 \mathrm{~ns}$ \\
\hline 40 & $198.86^{*}$ & $158.76^{*}$ & $4.62^{*}$ & $5.12^{*}$ & $20.26^{*}$ & $76.89^{*}$ \\
\hline 60 & $184.77^{*}$ & $147.23^{*}$ & $4.29^{*}$ & $4.29^{*}$ & $21.66^{*}$ & $71.74 \mathrm{~ns}$ \\
\hline 80 & $178.55^{*}$ & $135.55^{*}$ & $4.18^{*}$ & $4.37^{*}$ & $20.75^{*}$ & $65.96 \mathrm{~ns}$ \\
\hline 100 & $172.64^{*}$ & $124.78 \mathrm{~ns}$ & $3.38 \mathrm{~ns}$ & $3.68 \mathrm{~ns}$ & $16.56 \mathrm{~ns}$ & $60.47 \mathrm{~ns}$ \\
\hline
\end{tabular}

${ }^{*}$ Significant at $P \leq 0.05$, least means squares analysis; SME: sugar mill effluent.

TABLE 8: ANOVA for the effect of sugar mill effluent on concentrations of metals and biochemical components of $Z$. mays.

\begin{tabular}{lcccccccc}
\hline Source & $\mathrm{Zn}^{2+}$ & $\mathrm{Cd}^{2+}$ & $\mathrm{Cu}^{2+}$ & $\mathrm{Mn}^{2+}$ & $\mathrm{Cr}^{3+}$ & Crude proteins & Crude fiber & Total carbohydrates \\
\hline Season $(\mathrm{S})$ & $*$ & $*$ & $*$ & $*$ & $*$ & $*$ & $*$ & $*$ \\
SME concentration $(\mathrm{C})$ & $* *$ & $* *$ & $* *$ & $* *$ & $* *$ & $* *$ & $* *$ & $* *$ \\
Interaction S $\times \mathrm{C}$ & $* *$ & $* *$ & $* *$ & $* *$ & $* *$ & $* *$ & $* *$ & $* *$ \\
\hline
\end{tabular}

${ }^{*},{ }^{* *}$ : significant at $P \leq 0.05$ or $P \leq 0.01$, ANOVA; SME: sugar mill effluent.

carbohydrates were recorded with $40 \%$ sugar mill effluent concentrations in both seasons (Figures 4, 5, and 6). Content of crude proteins $(r=+0.21$ and $r=+0.34)$, crude fiber $(r=$ +0.20 and $r=+0.18)$, and total carbohydrates $(r=+0.06)$ were noted to be positively correlated with all concentrations of sugar mill effluent in both seasons. The contamination factor (Cf) was affected in both seasons (Figures 7-9). The $20 \%$ to $100 \%$ sugar mill effluent concentrations affected $\mathrm{Zn}^{2+}$, $\mathrm{Cd}^{2+}, \mathrm{Cu}^{2+}, \mathrm{Mn}^{2+}$, and $\mathrm{Cr}^{3+}$ contents in Z. mays. Increased irrigation frequency could lead to increase of metals in tissues. The $\mathrm{Zn}^{2+}, \mathrm{Cd}^{2+}, \mathrm{Cu}^{2+}, \mathrm{Mn}^{2+}$, and $\mathrm{Cr}^{3+}$ contents in $Z$. mays were noted highest with $100 \%$ sugar mill effluent (Figures 7 and 8). They were correlated with the content of $\mathrm{Zn}^{2+}, \mathrm{Cd}^{2+}, \mathrm{Cu}^{2+}, \mathrm{Mn}^{2+}$, and $\mathrm{Cr}^{3+}$ in $\mathrm{Z}$. mays and were positively correlated with sugar mill effluent concentrations in both seasons (Table 9). The Cf of various heavy metals was in the order of $\mathrm{Mn}^{2+}>\mathrm{Zn}^{2+}>\mathrm{Cu}^{2+}>\mathrm{Cr}^{3+}>$ $\mathrm{Cd}^{2+}$ in $Z$. mays after irrigation with sugar mill effluent (Figure 9). The highest contamination factor was noted for $\mathrm{Mn}^{2+}$; the least was observed for $\mathrm{Cd}^{2+}$ in $Z$. mays with $100 \%$ sugar mill effluent in both seasons. The contents of heavy metals were noted higher at higher concentrations of sugar mill effluent and likely inhibited the growth of $Z$. mays. The $40 \%$ concentration of sugar mill effluent was favored in vegetative growth, grain filling, and maturity of $Z$. mays. This is likely due to the optimal uptake of these metals by crop plants, which support various biochemical and physiological processes.

\section{Conclusions}

This study concluded that the sugar mill effluent increased the EC, OC, $\mathrm{Na}^{+}, \mathrm{K}^{+}, \mathrm{Ca}^{2+}, \mathrm{Mg}^{2+}, \mathrm{Fe}^{3+}, \mathrm{TKN}, \mathrm{PO}_{4}{ }^{3-}$, $\mathrm{SO}_{4}{ }^{2-}, \mathrm{Zn}^{2+}, \mathrm{Cd}^{2+}, \mathrm{Cu}^{2+}, \mathrm{Mn}^{2+}$, and $\mathrm{Cr}^{3+}$ of the soil in both the rainy and summer season. The effluent irrigation significantly changed the soil quality and affected the natural composition of the soil. Such alterations improved the fertility and enhanced the nutrient status of soil at lower concentrations of effluent irrigation. The accumulations of heavy metals, namely, $\mathrm{Cd}^{2+}, \mathrm{Cr}^{3+}, \mathrm{Cu}^{2+}, \mathrm{Mn}^{2+}$, and $\mathrm{Zn}^{2+}$, in soil and $Z$. mays were increased with an increase in sugar mill effluent concentrations in both seasons. These were recorded maximum with $100 \%$ concentration of sugar mill effluent. The agronomic performance of $Z$. mays was gradually increased from $20 \%$ to $40 \%$ and decreased from $60 \%$ to $100 \%$ concentration of sugar mill effluent in both seasons. The maximum growth performance of $Z$. mays was noted with $40 \%$ concentration of sugar mill effluent in both seasons. It may be due to the fact that, at lower concentrations, the nutrients and heavy metals may provide better and much 
TABLE 9: Coefficient of correlation $(r)$ between sugar mill effluent and $Z$. mays in both seasons.

\begin{tabular}{|c|c|c|}
\hline Effluent/Z.mays & Season & $r$-value \\
\hline \multirow{2}{*}{ Sugar mill effluent versus seed germination } & Rainy & -0.47 \\
\hline & Summer & -0.53 \\
\hline \multirow{2}{*}{ Sugar mill effluent versus RT } & Rainy & +0.74 \\
\hline & Summer & +0.73 \\
\hline \multirow{2}{*}{ Sugar mill effluent versus plant height } & Rainy & +0.10 \\
\hline & Summer & +0.07 \\
\hline \multirow{2}{*}{ Sugar mill effluent versus root length } & Rainy & +0.13 \\
\hline & Summer & -0.14 \\
\hline \multirow{2}{*}{ Sugar mill effluent versus number of leaves } & Rainy & +0.58 \\
\hline & Summer & +0.41 \\
\hline \multirow{2}{*}{ Sugar mill effluent versus fresh weight } & Rainy & +0.07 \\
\hline & Summer & +0.10 \\
\hline \multirow{2}{*}{ Sugar mill effluent versus dry weight } & Rainy & +0.05 \\
\hline & Summer & +0.03 \\
\hline \multirow{2}{*}{ Sugar mill effluent versus chlorophyll content } & Rainy & +0.16 \\
\hline & Summer & +0.28 \\
\hline \multirow{2}{*}{ Sugar mill effluent versus LAI } & Rainy & +0.50 \\
\hline & Summer & +0.50 \\
\hline \multirow{2}{*}{ Sugar mill effluent versus cob length } & Rainy & +0.32 \\
\hline & Summer & +0.33 \\
\hline \multirow{2}{*}{ Sugar mill effluent versus crop yield/plant } & Rainy & +0.05 \\
\hline & Summer & -0.15 \\
\hline \multirow{2}{*}{ Sugar mill effluent versus HI } & Rainy & +0.10 \\
\hline & Summer & +0.09 \\
\hline \multirow{2}{*}{ Sugar mill effluent versus $\mathrm{Zn}^{2+}$} & Rainy & +0.95 \\
\hline & Summer & +0.97 \\
\hline \multirow{2}{*}{ Sugar mill effluent versus $\mathrm{Cd}^{2+}$} & Rainy & +0.86 \\
\hline & Summer & +0.87 \\
\hline \multirow{2}{*}{ Sugar mill effluent versus $\mathrm{Cu}^{2+}$} & Rainy & +0.99 \\
\hline & Summer & +0.99 \\
\hline \multirow{2}{*}{ Sugar mill effluent versus $\mathrm{Mn}^{2+}$} & Rainy & +0.99 \\
\hline & Summer & +0.98 \\
\hline \multirow{2}{*}{ Sugar mill effluent versus $\mathrm{Cr}^{3+}$} & Rainy & +0.89 \\
\hline & Summer & +0.90 \\
\hline
\end{tabular}

effective stimulation to the agronomic performance of the $Z$. mays, while more irrigation increased the accumulation of nutrients/heavy metals at higher effluent concentrations (i.e., $60 \%$ and $100 \%$ ), thus inhibiting the overall performance of the crop plants. Biochemical components like crude proteins, crude fiber, and total carbohydrates were also highest with $40 \%$ sugar mill effluent in both seasons. The contamination factor (Cf) of various heavy metals was in the order of $\mathrm{Mn}^{2+}>$ $\mathrm{Zn}^{2+}>\mathrm{Cu}^{2+}>\mathrm{Cd}^{2+}>\mathrm{Cr}^{3+}$ for soil and $\mathrm{Mn}^{2+}>\mathrm{Zn}^{2+}>$ $\mathrm{Cu}^{2+}>\mathrm{Cr}^{3+}>\mathrm{Cd}^{2+}$ for $Z$. mays in both seasons after fertigation with sugar mill effluent. It appears that sugar mill effluent can be used as a biofertigant after appropriate dilution to improve the yield. Further studies on the agronomic growth and changes in biochemical composition of $Z$. mays after sugar mill effluent irrigation are required.

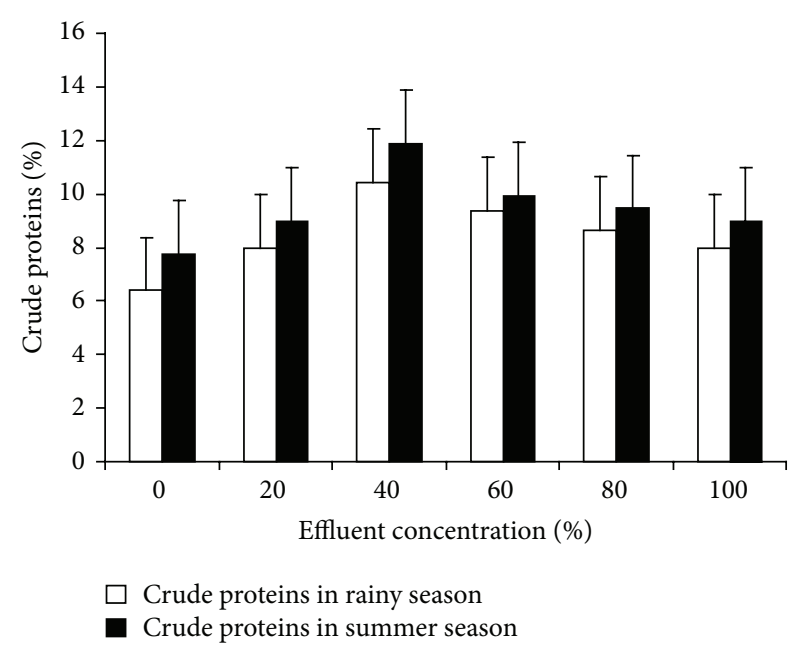

FIGURE 4: Crude proteins in Z. mays after irrigation with sugar mill effluent. Error bars are standard error of the mean.

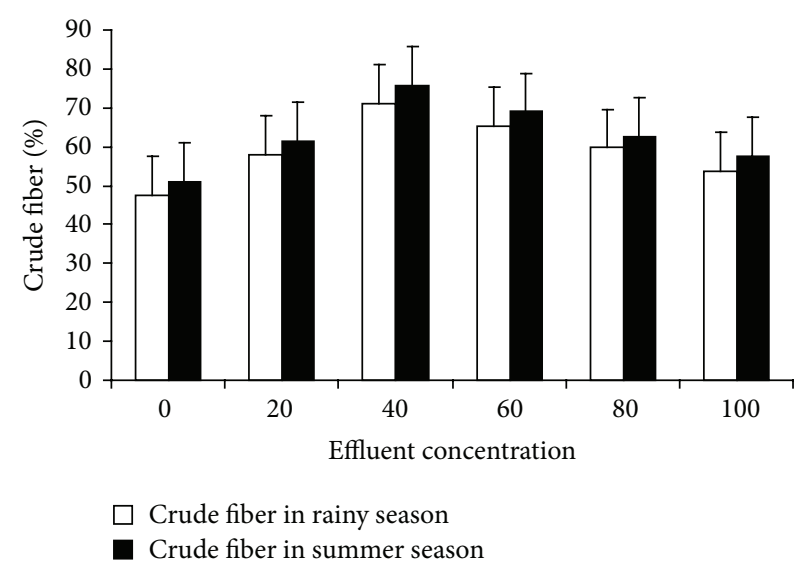

Figure 5: Crude fiber in Z. mays after irrigation with sugar mill effluent. Error bars are standard error of the mean.

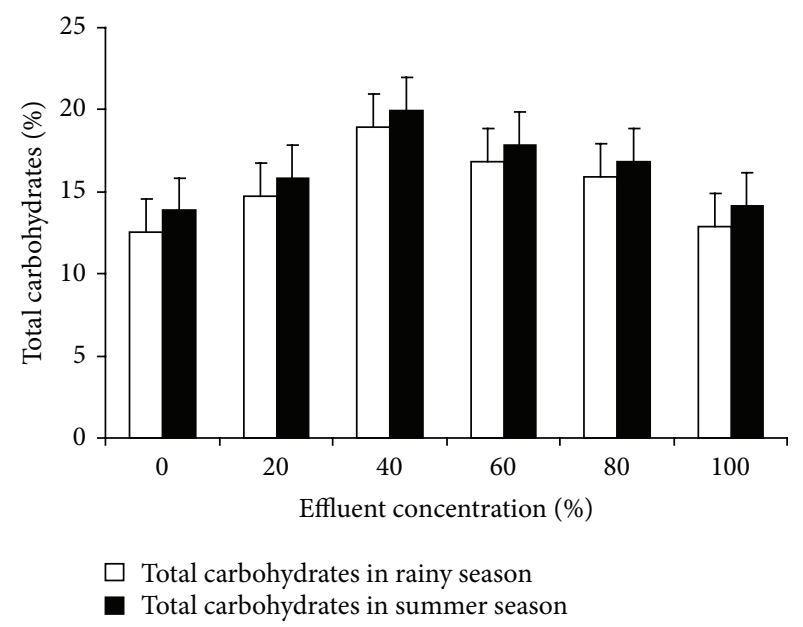

FIGURE 6: Total carbohydrates in Z. mays after irrigation with sugar mill effluent. Error bars are standard error of the mean. 


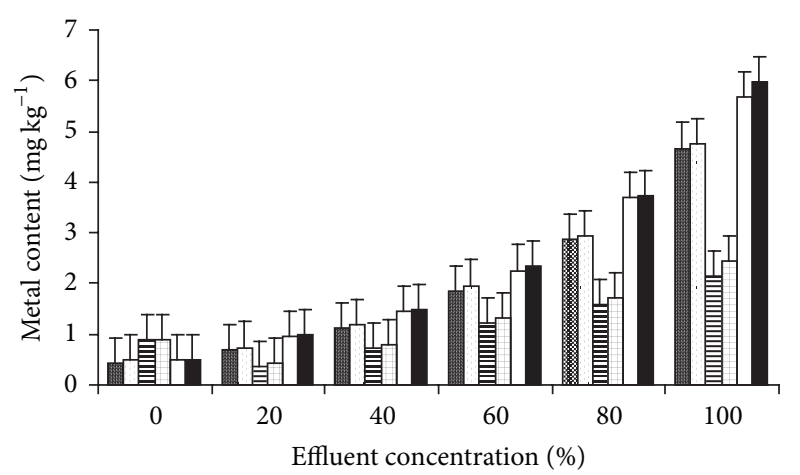

$\begin{array}{ll}\square \mathrm{Zn}^{2+} \text { in rainy season } & \square \mathrm{Zn}^{2+} \text { in summer season } \\ \text { 目 } \mathrm{Cd}^{2+} \text { in rainy season } & \square \mathrm{Cd}^{2+} \text { in summer season } \\ \square \mathrm{Mn}^{2+} \text { in rainy season } & \square \mathrm{Mn}^{2+} \text { in summer season }\end{array}$

FIGURE 7: Content of Zn, Cd, and Mn in Z. mays after irrigation with sugar mill effluent. Error bars are standard error of the mean.

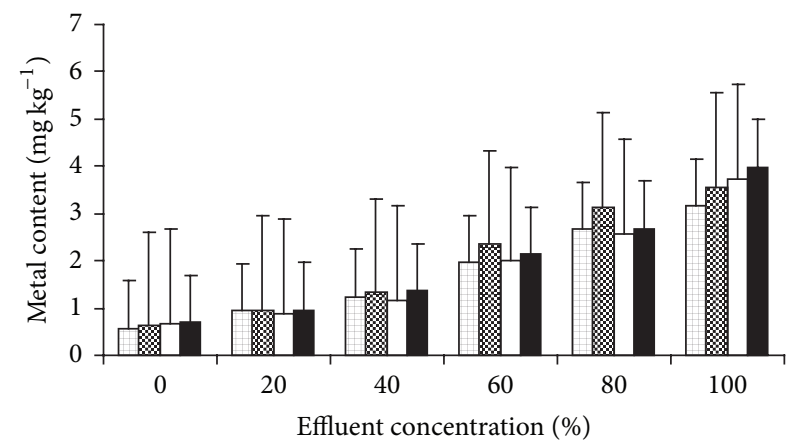

$$
\begin{array}{ll}
\square \mathrm{Cr}^{3+} \text { in rainy season } & \mathrm{Cr}^{3+} \text { in summer season } \\
\square \mathrm{Cu}^{2+} \text { in rainy season } & \mathrm{Cu}^{2+} \text { in summer season }
\end{array}
$$

Figure 8: Content of $\mathrm{Cr}$ and $\mathrm{Cu}$ in Z. mays after irrigation with sugar mill effluent. Error bars are standard error of the mean.

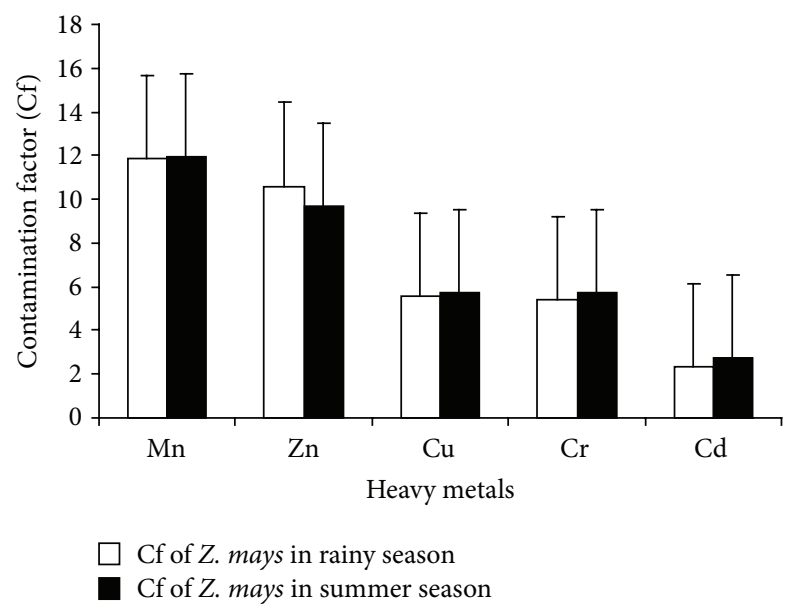

FIGURE 9: Contamination factor of heavy metals in Z. mays after irrigation with sugar mill effluent. Error bars are standard error of the mean.

\author{
Abbreviations \\ ANOVA: Analysis of variance \\ BD: Bulk density \\ BIS: $\quad$ Bureau of Indian standards \\ BWW: Bore well water \\ CD: $\quad$ Critical difference \\ Cf: Contamination factor \\ HI: $\quad$ Harvest index \\ LAI: $\quad$ Leaf area index \\ MPN: Most probable numbers \\ RT: $\quad$ Relative toxicity \\ SPC: $\quad$ Standard plate count \\ WHC: Water holding capacity.
}

\section{Conflict of Interests}

The author declares that there is no conflict of interests regarding the publication of this paper.

\section{Acknowledgments}

The University Grants Commission, New Delhi, India, is acknowledged for providing the financial support in the form of UGC research fellowship (F.7-70/2007-2009 BSR) to the corresponding author.

\section{References}

[1] A. R. A. Usman and M. A.-E. Gameh, "Effect of sugar industry wastes on $\mathrm{K}$ status and nutrient availability of a newly reclaimed loamy sandy soil," Archives of Agronomy and Soil Science, vol. 54, no. 6, pp. 665-679, 2008.

[2] T. A. Gemtos, N. Chouliaras, and S. Marakis, "Vinasse rate, time of application and compaction effect on soil properties and durum wheat crop," Journal of Agricultural Engineering Research, vol. 73, no. 3, pp. 283-296, 1999.

[3] K. Arindam and A. B. Prasad, "Alteration in physico-chemical characteristics of soil under stress of carbonaceous sugar mill," Advances in Plant Science, vol. 12, pp. 103-109, 1999.

[4] D. Ezhilvannan, P. S. Sharavanan, and M. Vijayaragavan, "Effect of sugar mill effluent on changes of growth and amino acid and protein contents of maize (Zea mays L.) plants," Journal of Ecobiotechnology, vol. 3, no. 7, pp. 26-29, 2011.

[5] N. P. Rathore, S. A. Iqbal, and K. S. Pawan, "Role of sugar industry effluent in agriculture," Indian Journal of Applied \& Pure Biology, vol. 19, pp. 91-94, 2000.

[6] A. Almodares and M. E. Sharif, "Effects of irrigation water qualities on biomass and sugar contents of sugar beet and sweet sorghum cultivars," Journal of Environmental Biology, vol. 28, no. 2, pp. 213-218, 2007.

[7] A. K. Baruah, R. N. Sharma, and G. C. Borah, "Impact of sugar mill and distillery effluent on water quality of river Galabil," Assam, Indian Journal of Environmental Health, vol. 35, pp. 288293, 1993.

[8] S. M. Arafat, "Evaluation of sugar cane filter mud cake on improving soil characteristics and water melon yield," Egyptian Journal of Applied Sciences, vol. 9, pp. 287-295, 1994.

[9] A. A. Yassen, S. M. Arafat, and S. M. Zaghloul, "Maximizing use of vinasse and filter mud as byproducts of sugar cane on 
wheat productions," Journal of Agricultural Science, Mansoura University, vol. 27, pp. 7865-7873, 2002.

[10] M. A. Khan, S. Shahid Shaukat, A. Shahzad, and H. Arif, "Growth and yield responses of pearl millet (Pennisetum glaucum [L.] R.Br.) irrigated with treated effluent from waste stabilization ponds," Pakistan Journal of Botany, vol. 44, no. 3, pp. 905-910, 2012.

[11] A. U. Osaigbovo and E. R. Orhue, "Influence of pharmaceutical effluent on some soil chemical properties and early growth of maize (Zea mays L)," African Journal of Biotechnology, vol. 5, no. 18, pp. 1612-1617, 2006.

[12] V. Kumar and A. K. Chopra, Effect of industrial effluent irrigation on agronomical characteristics of two leguminous crops, Phaseolus vulgaris (L.) and Vigna radiata (L.) [Ph.D. thesis], Department of Zoology and Environmental Science, Gurukula Kangri University, Haridwar, India, 2010.

[13] V. Kumar and A. K. Chopra, "Fertigation effect of distillery effluent on agronomical practices of Trigonella foenum-graecum L. (Fenugreek)," Environmental Monitoring and Assessment, vol. 184, no. 3, pp. 1207-1219, 2012.

[14] H. L. Frost and L. H. Ketchum Jr., "Trace metal concentration in durum wheat from application of sewage sludge and commercial fertilizer," Advances in Environmental Research, vol. 4, no. 4, pp. 347-355, 2000.

[15] F. Itanna, "Metals in leafy vegetables grown in Addis Ababa and toxicological implications," Ethiopian Journal of Health Development, vol. 6, pp. 295-302, 2002.

[16] K. Marr, H. Fyles, and W. Hendershot, "Trace metals in montreal urban soils and the leaves of Taraxacum officinale," Canadian Journal of Soil Science, vol. 79, no. 2, pp. 385-387, 1999.

[17] A. K. Chopra, C. Pathak, and G. Parasad, "Scenario of heavy metal contamination in agricultural soil and its management," Journal of Applied and Natural Science, vol. 1, no. 1, pp. 99-108, 2009.

[18] E. J. Ferguson, "Heavy metals in plants," in The Heavy Elements, Chemistry, Environmental Impact and Health Effects, E. J. Ferguson, Ed., pp. 7-30, Pergamon Press, Oxford, UK, 1990.

[19] K. M. Hati, A. K. Biswas, K. K. Bandyopadhyay, and A. K. Misra, "Soil properties and crop yields on a vertisol in India with application of distillery effluent," Soil and Tillage Research, vol. 92, no. 1-2, pp. 60-68, 2007.

[20] F. R. Boone and B. W. Veen, "The influence of mechanical resistance and phosphate supply on morphology and function of maize roots," Netherlands Journal of Agricultural Science, vol. 30, pp. 179-192, 1982.

[21] W. S. Meyer, H. D. Barrs, A. R. Mosier, and N. L. Schaefer, "Response of maize to three short-term periods of waterlogging at high and low nitrogen levels on undisturbed and repacked soil," Irrigation Science, vol. 8, no. 4, pp. 257-272, 1987.

[22] M. O. Akande, E. A. Makinde, and M. T. Adetunji, "Response of maize and cowpea grown sequentially to application of phosphate rock in the humid tropics," Communications in Soil Science and Plant Analysis, vol. 42, no. 9, pp. 1027-1037, 2011.

[23] C. R. Boomsma, J. B. Santini, T. D. West, J. C. Brewer, L. M. McIntyre, and T. J. Vyn, "Maize grain yield responses to plant height variability resulting from crop rotation and tillage system in a long-term experiment," Soil and Tillage Research, vol. 106, no. 2, pp. 227-240, 2010.

[24] C. Mansouri-Far, S. A. M. Modarres Sanavy, and S. F. Saberali, "Maize yield response to deficit irrigation during low-sensitive growth stages and nitrogen rate under semi-arid climatic conditions," Agricultural Water Management, vol. 97, no. 1, pp. 12-22, 2010.

[25] S. A. Materechera and H. R. Mloza-Banda, "Soil penetration resistance, root growth and yield of maize as influenced by tillage system on ridges in Malawi," Soil and Tillage Research, vol. 41, no. 1-2, pp. 13-24, 1997.

[26] A. Kaushik, B. R. Kadyan, and C. P. Kaushik, "Sugarmill effluent effects on growth, photosynthetic pigments and nutrient uptake in wheat seedlings in aqueous vs. soil medium," Water, Air, and Soil Pollution, vol. 87, no. 1-4, pp. 39-46, 1996.

[27] R. N. Bharagava, R. Chandra, and V. Rai, "Phytoextraction of trace elements and physiological changes in Indian mustard plants (Brassica nigra L.) grown in post methanated distillery effluent (PMDE) irrigated soil," Bioresource Technology, vol. 99, no. 17, pp. 8316-8324, 2008.

[28] J. Mendoza, T. Garrido, G. Castillo, and N. S. Martin, "Metal availability and uptake by sorghum plants grown in soils amended with sludge from different treatments," Chemosphere, vol. 65, no. 11, pp. 2304-2312, 2006.

[29] N. El Sawaf, "Response of Sorghum spp. to sewage waste-water irrigation," International Journal of Agriculture and Biology, vol. 7, no. 6, pp. 869-874, 2005.

[30] T. D. West, D. R. Griffith, G. C. Steinhardt, E. J. Kladivko, and S. D. Parsons, "Effect of tillage and rotation on agronomic performance of corn and soybean: twenty-year study on dark silty clay loam soil," Journal of Production Agriculture, vol. 9, no. 2, pp. 241-248, 1996.

[31] W. W. Wilhelm, J. M. F. Johnson, J. L. Hatfield, W. B. Voorhees, and D. R. Linden, "Crop and soil productivity response to corn residue removal: a literature review," Agronomy Journal, vol. 96, no. 1, pp. 1-17, 2004.

[32] APHA, Standard Methods for the Examination of Water and Wastewater, American Public Health Association, Washington, DC, USA, 21st edition, 2005.

[33] R. K. Chaturvedi and K. Sankar, Laboratory Manual for the Physico-Chemical Analysis of Soil, Water and Plant, Wildlife Institute of India, Dehradun, India, 2006.

[34] L. Radhouane, "Salinity effect on germination, growth, and grain production of some autochthonous pear millet ecotypes (Pennisetum glaucum (L.) R. Br.)," Comptes Rendus Biologies, vol. 331, no. 4, pp. 278-286, 2008.

[35] C. Milner and R. E. Hughes, "Methods for the measurement of primary production of grassland," in IBP Handbook no. 6, Blackwell Scientific Publications, Oxford, UK, 1968.

[36] R. J. Porra, "The chequered history of the development and use of simultaneous equations for the accurate determination of chlorophylls a and b," Photosynthesis Research, vol. 73, no. 1-3, pp. 149-156, 2002.

[37] N. Chapagain, Physiological impact of Dhobikhola (Kathmandu) water pollution on Persicaria perfoliata L. leaves and germination of some vegetable seeds [M.S. thesis], Central Department of Botany, Tribunal University, Kathmandu, Nepal, 1991.

[38] R. F. Denison and R. Russotti, "Field estimates of green leaf area index using laser-induced chlorophyll fluorescence," Field Crops Research, vol. 52, no. 1-2, pp. 143-149, 1997.

[39] T. R. Sinclair, "Historical changes in harvest index and crop nitrogen accumulation," Crop Science, vol. 38, no. 3, pp. 638643, 1998.

[40] Anonymous, Official Methods of Analysis, Association of Official Analytical Communities, Washington, DC, USA, 1980. 
[41] J. Cerning and J. Guilhot, "Changes in carbohydrates composition during maturation of wheat and barley kernel," Cereal Chemistry, vol. 50, pp. 220-224, 1973.

[42] L. Hakanson, "An ecological risk index for aquatic pollution control. A sedimentological approach," Water Research, vol. 14, no. 8, pp. 975-1001, 1980.

[43] BIS, "Indian standards for drinking water-specification (BIS 10500:1991)," 1991, http://www.bis.org.in/index.asp.

[44] D. D. Borole and P. R. Patil, "Studies on physico-chemical parameters and concentration of heavy metals in sugar industry," Pollution Research, vol. 23, no. 1, pp. 83-86, 2004.

[45] A. K. Biswas, M. Mohanty, K. M. Hati, and A. K. Misra, "Distillery effluents effect on soil organic carbon and aggregate stability of a Vertisol in India," Soil and Tillage Research, vol. 104, no. 2, pp. 241-246, 2009.

[46] L. Baskaran, G. K. Sankar, A. L. A. Chidambaram, and P. Sundaramoorthy, "Amelioration of sugar mill effluent polluted soil and its effect of green gram (Vigna radiata L.)," Botany Research International, vol. 2, no. 2, pp. 131-135, 2009.

[47] P. E. V. Charman and B. W. Murphy, Soils: Their Properties and Management: A Soil Conservation Handbook for New South Wales, Sydney University Press, Sydney, Australia, 1991.

[48] A. M. Azza Mazher, E. L. Fatma, E. M. Quensi, and M. M. Farahat, "Responses of ornamental plants and woody trees to salinity," World Journal of Agricultural Sciences, vol. 3, no. 3, pp. 386-395, 2007.

[49] A. K. Chopra, S. Srivastava, V. Kumar, and C. Pathak, "Agropotentiality of distillery effluent on soil and agronomical characteristics of Abelmoschus esculentus L. (okra)," Environmental Monitoring and Assessment, vol. 185, no. 8, pp. 6635-6644, 2013.

[50] O. A. Al-Tahir, Y. A. Al-Nabulsi, and A. M. Helalia, "Effects of water quality and frequency of irrigation on growth and yield of barley (Hordeum vulgate L.)," Agricultural Water Management, vol. 34, no. 1, pp. 17-24, 1997.

[51] G. Abdel-Nasser and S. M. El-Shazly, "Response of Picual olive trees to potassium and boron fertigation. 1-Vegetative growth and leaf constituents," Journal of Advertising Research, vol. 6, pp. 631-649, 2001.

[52] N. M. Zalawadia and S. Raman, "Effect of distillery wastewater with graded fertilizer levels on sorghum yield and soil properties," Journal of the Indian Society of Soil Science, vol. 42, pp. 575579, 1994.

[53] A. H. El-Naggar, "Effect of foliar nutrition on growth, flowering, corms and cormels production of gladiolus plants," Alexandria Science Exchange, vol. 26, no. 1, pp. 19-27, 2005.

[54] S. N. Pandey, B. D. Nautiyal, and C. P. Sharma, "Pollution level in distillery effluent and its phytotoxic effect on seed germination and early growth of maize and rice," Journal of Environmental Biology, vol. 29, no. 2, pp. 267-270, 2008.

[55] M. Naeem, J. Iqbal, and B. Maaha, "Comparative study of inorganic fertilizers and organic manures on yield and yield components of Mung bean (Vigna radiata L.)," Journal of Agriculture and Social Science, vol. 2, no. 4, pp. 227-229, 2006. 

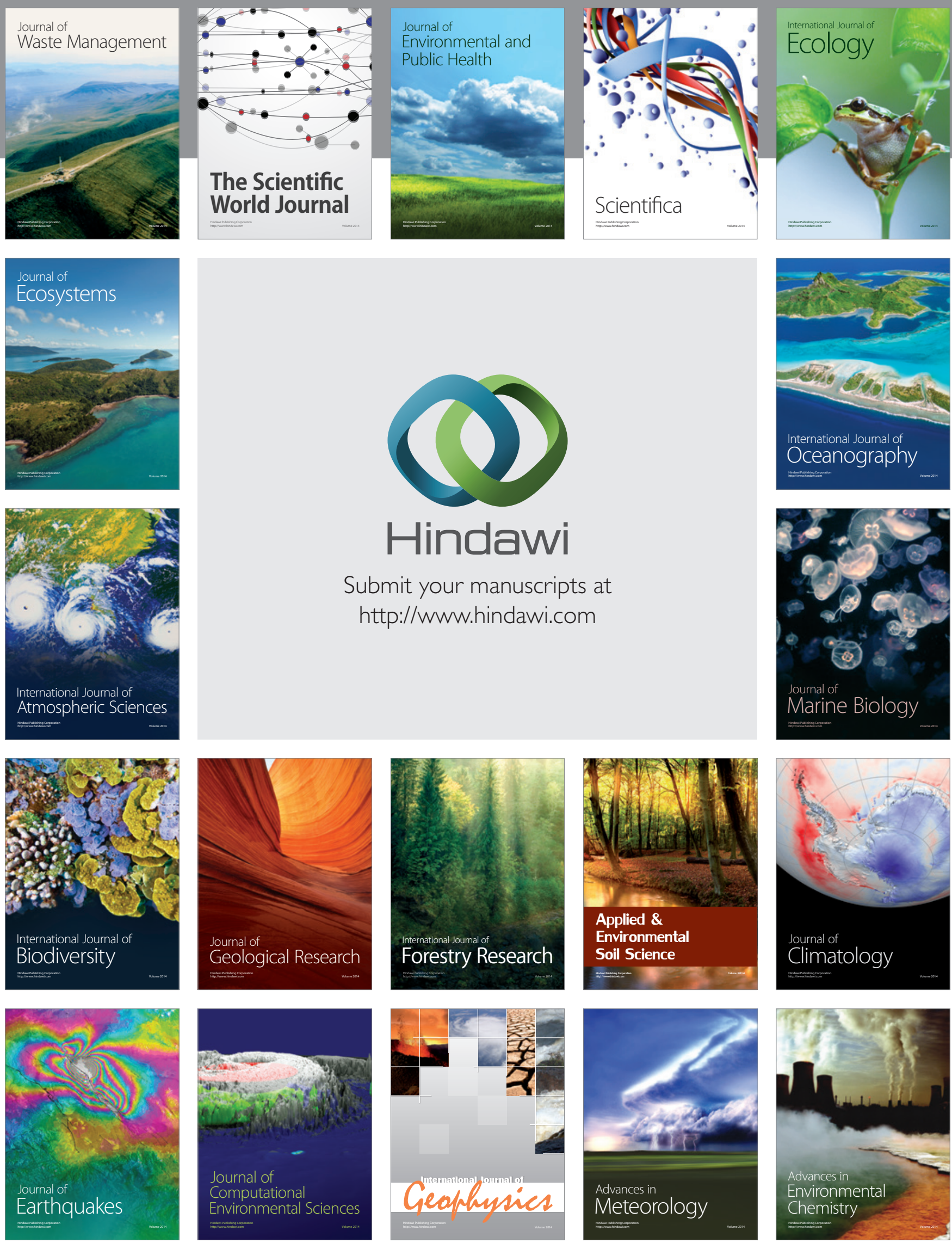